\title{
Effects of juvenile hormone analogue (methoprene) and 20-hydroxyecdysone on reproduction in Polygonia c-aureum (Lepidoptera: Nymphalidae) in relation to adult diapause
}

\section{Authors: Satoshi Hiroyoshi, Gadi V. P. Reddy, and Jun Mitsuhashi}

The final publication is available at Springer via http://dx.doi.org/10.1007/s00359-017-1179-3.

Hiroyoshi, Satoshi, Gadi V. P. Reddy, and Jun Mitsuhashi. "Effects of juvenile hormone analogue (methoprene) and 20-hydroxyecdysone on reproduction in Polygonia c-aureum (Lepidoptera: Nymphalidae) in relation to adult diapause." Journal of Comparative Physiology A (August 2017): 1-13. DOI: 10.1007/s00359-017-1179-3.

Made available through Montana State University's $\underline{\text { ScholarWorks }}$ scholarworks. montana.edu 


\title{
Effects of juvenile hormone analogue (methoprene) and 20-hydroxyecdysone on reproduction in Polygonia c-aureum (Lepidoptera: Nymphalidae) in relation to adult diapause
}

\author{
Satoshi Hiroyoshi ${ }^{1,2} \cdot$ Gadi V. P. Reddy $^{3} \cdot J^{\prime}$ Mitsuhashi ${ }^{1,4}$
}

\begin{abstract}
We investigated the effects of juvenile hormone analogue (methoprene) and 20-hydroxyecdysone on female and male reproduction in a nymphalid butterfly, Polygonia c-aureum. This butterfly has a facultative adult diapause controlled by the corpora allata and brain. Methoprene seems to terminate reproductive diapause, although transplantation experiments indicate that the activity of the corpora allata does not affect male mating behavior Endo (Dev Growth Differ 15:1-10, 1973a), suggesting that the brain may be involved in diapause. We found that exposure to methoprene promoted the development of ovaries and of the male accessory glands and simplex. On the other hand, exposure to 20-hydroxyecdysone did not promote the development of female and male reproductive organs and eupyrene sperm movement from the testis to the duplex in the adult stage. Ecdysteroid titer in both sexes was consistently low in adults. These results suggest that imaginal diapause is largely regulated by juvenile hormone in this butterfly.
\end{abstract}

Keywords Accessory gland - Corpora allata $\cdot$ Ecdysteroid . Testis · Simplex

Satoshi Hiroyoshi

satoshi_hiroyoshi@yahoo.co.jp

1 Laboratory of Applied Entomology, Faculty of Agriculture, Tokyo University of Agriculture and Technology, Fuchu, Tokyo 183-0054, Japan

2 Kotobukiso 7-12-203, Nisikawa-cho, Itoman, Okinawa 901-0304, Japan

3 Western Triangle Ag Research Center, Montana State University, 9546 Old Shelby Rd., P. O. Box 656, Conrad, MT 59425, USA

4 1-28-13 Koishikawa, Bunkyouku, Tokyo 112-0002, Japan

\author{
Abbreviations \\ CA Corpus allatum \\ JH Juvenile hormone \\ MAG Male accessory gland \\ AGPs Accessory gland products \\ JHA Juvenile hormone analogue \\ PTTH Prothoracicotropic hormone \\ LD Long daylength \\ SD Short daylength \\ RIA Radioimmunoassay \\ 20E 20-hydroxyecdysone \\ NPF Neuropeptide F
}

\section{Introduction}

Inactivity of the corpus allatum (CA) has been found to be a typical feature of adult diapause (Hodková 1977a; Tauber et al. 1986; De Kort et al. 1987; Taub-Montemayor and Rankin 1997). A decline or disappearance of hemolymph juvenile hormone(s) $(\mathrm{JH})$ due to inactivity of the CA triggers various diapause symptoms, including the inhibition of ovarian development, the reduction of the synthesis of accessory gland substances, and the development of fat bodies in females (Hodková 1977b; Duportets et al. 1998; Socha et al. 2004). Male adult diapause is also generally induced by inactivity of the CA (Pener 1992). Thus, inactivity of the CA induces and maintains diapause by causing a decline or disappearance of $\mathrm{JH}$ in hemolymph. Conversely, topical application of $\mathrm{JH}$ was found to break diapause in the Colorado potato beetle, Leptinotarsa decemlineata Say (Schooneveld et al. 1977). The CA produces and secretes JHs, which provoke almost all physiological, developmental, and reproductive processes (Khalil Sayed et al. 2006; Jindra et al. 2013; Sláma 2015). These processes are 
controlled by the synthesis, degradation, sequestration, and secretion of JH (Gilbert et al. 2000).

Secretions from the male accessory gland (MAG) contain a variety of bioactive molecules (Gillott 1996, 2003). Juvenile hormones regulate the secretory activity of MAG (Chen 1984). In several species of moths, JH or JH acid is stored in the male accessory glands and transferred to females during copulation (Shirk et al. 1976, 1980; Pszczolkowski et al. 2006; Hassanien et al. 2014; Clifton et al. 2014; Paroulek and Sláma 2014). Sex peptides derived from accessory glands stimulate the female's CA to synthesize and release $\mathrm{JH}$, promoting oocyte maturation via a series of endocrine processes (Richard et al. 1998). The synthesis of JH by MAG is not confined to Lepidoptera (Davey 2000). In the mosquito Aedes aegypti (L.) (Diptera), JH synthesized by the male CA is used for internal endocrinological regulation, while $\mathrm{JH}$ synthesized by the MAG is transferred to females (Borovsky et al. 1994). The major components of MAG secretions are proteins, collectively named accessory gland products (AGPs) (Ram and Ramesh 2003). Upon copulation, the transfer of AGPs has been shown in many insects to trigger profound physiological and behavioral changes in females, including enhanced ovulation, oviposition, cessation of pheromone production, and reduced mating receptivity (Dottorini et al. 2007). On the other hand, the inhibition of female sexual receptivity of the Mexican fruit fly Anastrepha ludens (Loew) was found to be mediated by factors other than AGPs, such as the number of sperm stored by females (Abraham et al. 2014)

In males of the Asian comma butterfly, Polygonia c-aureum L., adult diapause is characterized by inhibition of the development of the male accessory gland, the simplex, and mating behavior, as well as increased development of the fat body (Endo 1973a; Hiroyoshi unpublished data). This butterfly has seasonal forms, with the summerand autumn-forms being controlled by photoperiod and temperature during the larval and/or pupal stages. Summerform butterflies emerge in summer and quickly reproduce soon with several repeating non-diapausing generations in the warmer regions of Japan. In contrast, the autumnform butterflies that emerge in September through October enter adult diapause and reproduce in the following spring. Although autumn-form males and females that are in diapause have inactive corpus allata (Endo 1973a, b), whether diapause features are directly evoked by CA inactivity has not been demonstrated. Therefore, we examined the effects of a juvenile hormone analogue (JHA), methoprene, on the development of the reproductive organs of both sexes of $P$. c-arureum.

Molting and metamorphosis in insects are controlled by ecdysteroids from the prothoracic glands, whose secretion is regulated by the prothoracicotropic hormone $(\mathrm{PTTH})$ from the brain and insulin-like peptides in response to nutritional signals (Jindra et al. 2013). Post-embryonic development is highly dependent on two types of lipophilic hormones: ecdysteroids and JHs (Marchal et al. 2012). Ecdysteroid level increases as adult females mature, and its synthesis has been shown to be associated with the maturation of terminal oocytes in the house cricket, Acheta domesticus (L.) (Dinan 1997). Ecdysteroids also modulate male courtship behavior in the common fruit fly, Drosophila melanogaster Meigen (Ganter et al. 2011). It is also known that in the cricket Gryllus bimaculatus De Geer (Hoffmann and Behrens 1982) and in the blow fly Calliphora vicina Robineau-Desvoidy (Koolman et al. 1979) testes in adult males contain relatively large amounts of ecdysteroids compared with other organs or tissues. Gillott and Ismail 1995 demonstrated that male accessory glands, testes, abdominal integuments, and adhering fat body secrete ecdysteroids in in vitro cultures. Although the function of imaginal ecdysteroids is not well understood, ecdysteroid titer typically rises in adult insects before they enter diapause, such as has been shown in L. decemlineata (Briers and De Loof 1981; Briers et al. 1982). In the leaf beetle Gastrophysa atrocyanea Motschulsky, it has been suggested that ecdysteroids play an important role in the formation of diapause proteins (Ichimori et al. 1990) and, for D. melanogaster, that they are involved in the termination of imaginal diapause (Richard et al. 2001). However, the effects of ecdysteroids on ovarian development of lepidopteran adults is unclear. Thus, we also examined ecdysteroid titers and their effects on the reproduction of both sexes of P. c-aureum.

The simplex or ejaculatory duct is the largest organ in the male's reproductive tracts and constitutes the outer surface structure of the spermatophore in lepidopterans. Substances derived from the accessory gland, duplex (=seminal vesicle), the vas deferens, and testis are incorporated into the spermatophore. In this study, therefore, we also examined the effects of two hormones on the development of the accessory gland, testis and simplex.

\section{Materials and methods}

\section{Insect cultures}

Larvae of $P$. c-aureum were collected in a field in the Tokyo metropolitan area and in Saitama Prefecture in the central Honshu (Japan) and reared in the laboratory under long daylength (LD) conditions (15L:9D) at $21 \pm 1{ }^{\circ} \mathrm{C}$. Only individuals from eggs laid by summer-form adults were used for experiments, as offspring of autumn-form females are likely to become the summer-form under any photoperiodic conditions due to a maternal effect (Hidaka 
and Takahashi 1967). Eggs were surface-sterilized with a $3 \%$ formaldehyde solution for $30 \mathrm{~min}$, washed in tap water, air-dried, and then placed in a plastic Petri dish $(9 \mathrm{~cm}$ dia $\times 2 \mathrm{~cm}$ deep) until hatched. Newly emerged larvae were reared under either LD or SD conditions (short daylength $=8 \mathrm{~L}: 16 \mathrm{D}$ ) at $21 \pm 1{ }^{\circ} \mathrm{C}$, which caused them to produce summer- and autumn-form offspring, respectively. Larvae were reared in groups of 30-40 on filter paper in a glass Petri dish $(12 \mathrm{~cm}$ dia $\times 3 \mathrm{~cm}$ deep or $15 \mathrm{~cm}$ dia $\times 4 \mathrm{~cm}$ deep) containing fresh leaves of Japanese hops, Humulus japonicus Siebold and Zucc. (Moraceae). The larval density in a Petri dish was reduced after larvae became 4th instar. After adult emergence, females and males were held in separate cages $(17 \times 16.5 \times 46 \mathrm{~cm})$ covered with Saran ${ }^{\circledR}$ net in groups of $30-40$. Adults were fed $10 \%$ sugar solution ad libitum absorbed on cotton.

Two or three summer-form adults that had been reared under LD conditions for their entire life, or autumn-form adults that had been reared under SD conditions during their immature stages and then kept under LD or SD conditions after emergence until 30 days old, were used to measure ecdysteroid titers under these regimes.

\section{Determination of hemolymph ecdysteroid titers by RIA (Radioimmunoassay)}

Hemolymph ecdysteroid titers were measured using the method of Wani et al. (1997). The dorsal part of abdomens of adults of the various ages was pierced with forceps and hemolymph was collected. Samples of about 2 or $3 \mu \mathrm{l}$ of hemolymph were placed in a tube with 60 or $90 \mu \mathrm{l}$ of $100 \%$ methanol, respectively, and then centrifuged at $2500 \mathrm{~g}$ for $20 \mathrm{~min}$ (at $4{ }^{\circ} \mathrm{C}$ ), and the resulting supernatant was subjected to RIA (Bollenbacher et al. 1981). The antibody, Horn-22 serum (Horn et al. 1976), used in this experiment showed cross-reactivity with both ecdysone and 20-hydroxyecdysone (20E) at a ratio of 1:4.5 (Warren and Gilbert 1986). The ecdysteroid titer was expressed in $20 \mathrm{E}$ equivalents, since $20 \mathrm{E}$ was used as the radiolabeled ligand in the RIA.

\section{Hormone treatment}

Autumn-form butterflies that had been reared under SD photoperiod at $21 \pm 1{ }^{\circ} \mathrm{C}$ were used in the experiments to assess the effects of hormone on the female and male reproductive development in relation to adult diapause. Two days after emergence, control butterflies were treated with hormone or solvent by smearing or injection. Treated and control butterflies were anesthetized with $\mathrm{CO}_{2}$ in a polythene bag before treatment with solvent. A no-solvent, untreated second control group was neither anesthetized nor treated with solvent.
Juvenile hormone analogue treatment was done as per Wu et al. (1987). A JH analogue, methoprene (ZR515, Zoecon), was dissolved in acetone to provide stock solutions of 1 and $10 \mu \mathrm{g} / \mu \mathrm{l}$. After butterflies were anesthetized, $5 \mu \mathrm{l}$ samples (being 5 or $10 \mu \mathrm{g}$ of active ingredient) were applied topically to the ventral side of the abdomen. The same volume of acetone was applied as control. In the untreated group, anesthesia and application of solvent were not performed. Butterflies were dissected $5,10,20$, or 30 days after emergence to assess the reproductive tract for signs of diapause.

To provide stock solutions ( 1 or $10 \mu \mathrm{g} / \mu \mathrm{l})$ of 20-hydroxyecdysone (20E), we diluted concentrated material appropriately in $10 \%$ ethanol. After anesthetizing a butterfly, a $1-\mu 1$ sample (being 1 or $10 \mu \mathrm{g}$ of active ingredient) was injected into the hemocoele using a Hamilton microsyringe. The same volume of $10 \%$ methanol was applied as a control. In a second, untreated control group, butterflies were neither anesthetized nor injected with $10 \%$ methanol solvent. In another treatment, both methoprene and 20E were applied together, each as described above. Butterflies were dissected in groups, at 5 or 10 days after emergence.

\section{Effects of methoprene and $20 \mathrm{E}$ on reproduction}

The single testis formed by the fusion of two testes at the prepupal stage (Hiroyoshi 2016) was dissected out in saline solution $\left(8.6 \mathrm{~g} \mathrm{NaCl}, 0.33 \mathrm{~g} \mathrm{CaCl}_{2}\right.$, and $0.1 \mathrm{~g} \mathrm{KCl}$ per liter distilled water). The sample size for treatments ranged from 11 to 54. The length (L) and width (W) of the testis was measured with the aid of a calibrated ocular micrometer under a phase-microscope. Testis volume was calculated as volume $=\pi / 6 \times \mathrm{L} \times \mathrm{W}^{2}$, assuming that the testis to be an ideal ellipsoid (Nishiitsutusji-Uwo 1959). In order to determine the development of male reproductive organs, the dry mass of a pair of accessory glands and the simplex was measured at each designated age. Of the two types of sperm (eupyrene and apyrene) found in Lepidoptera (Hiroyoshi 1999), we counted the number of eupyrene sperm bundles in the duplex to assess the dynamics of sperm movement from the testis to the duplex via vasa deferentia on day 10 under a phase-microscope.

Ovaries were dissected out in a saline solution under a binocular microscope 5 or 10 days after adult eclosion. Diapausing females had small opaque or white-colored oocytes. Females with yellow, yellowish green, green oocytes or green eggs were non-diapausing butterflies. The number of eggs for each ovary was counted. The diameter of the largest oocyte in each butterfly was measured with the aid of an ocular micrometer equipped with a phase-contrast microscope. 


\section{Statistical analysis}

Data were analyzed by ANOVA, followed by Tukey's method of mean separation, or by Mann-Whitney $U$ test for ecdysteroid titer, testis size, mass of accessory glands (g) and simplex, the number of eggs, oocyte diameter, and the number of eupyrene sperm bundles in the duplex.

\section{Results}

\section{Determination of ecdysteroid titer}

We found no significant differences in male hemolymph ecdysteroid titer among summer-form butterflies reared under LD and autumn-form butterflies reared under SD or LD over the course of the adult stage (0-30 days old) $(F=4.553, d f=2, P>0.05)$ except in 20-day-old males (Fig. 1). Similar results were found for females (Fig. 2). We also found no significant differences between males and females at each particular age $(P>0.05)$ except for 15- and 17-day-old adults. Significant differences in ecdysteroid titers were detected between summerand autumn-form males by Mann-Whitney Utest (U1value $=46170, U 2$-value $=26730, W$ value $=82755$, $P<0.001)$ and between the autumn-form females reared under SD and LD after emergence $(U 1$-value $=25056$, $U 2$-value $=43065, W$ value $=59247, P<0.0001)$. However, we found no significant differences in ecdysteroid titer between males and females by Mann-Whitney $U$ test in either summer-form ( $U 1$-value $=16470$, $U 2$-value $=17019, W$ value $=33306, P=0.7812)$ or autumn-form $(U 1$-value $=61596, U 2$-value $=59508, W$ value $=122322, P=0.6867$ ) butterflies .

The average values of ecdysteroid titer in all individuals were $8.8 \mathrm{ng} / \mathrm{ml}$ in the summer-form, $12.8 \mathrm{ng} /$ $\mathrm{ml}$ in the autumn-form under $\mathrm{LD}$, and $7.3 \mathrm{ng} / \mathrm{ml}$ in the autumn-form under SD. Under all three rearing conditions, ecdysteroid titers in $P$. c-aureum adults were consistently low throughout the adult stage and there were many individuals showing a titer below a measurable level.
Fig. 1 Fluctuation of hemolymph ecdysteroid titer in adult male Polygonia c-aureum with age. LDS, LDA or SDA indicates summer-form adults reared under long-day conditions and autumn-form adults reared under short day in the immature stages and then kept under LD or SD after emergence, respectively

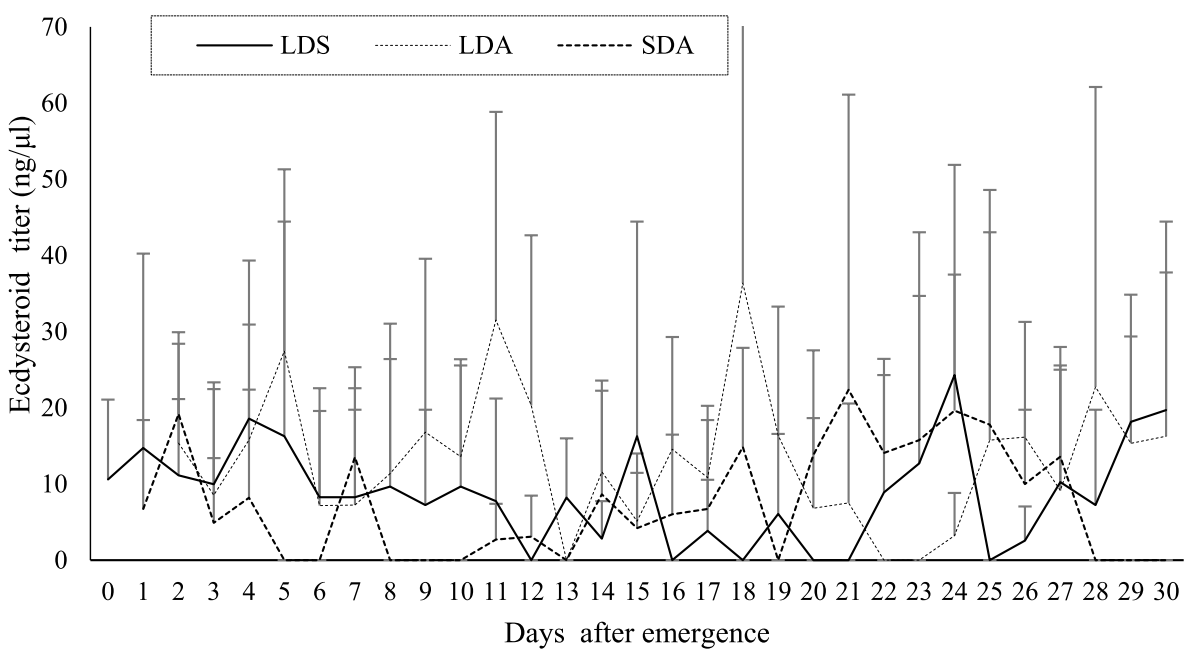

Fig. 2 Fluctuation of hemolymph ecdysteroid titer in adult female Polygonia c-aureum with age. LDS, LDA or SDA indicates summer-form adults reared under long-day conditions, and autumn-form adults reared under short-day conditions in the immature stages and then kept under LD or SD after emergence, respectively

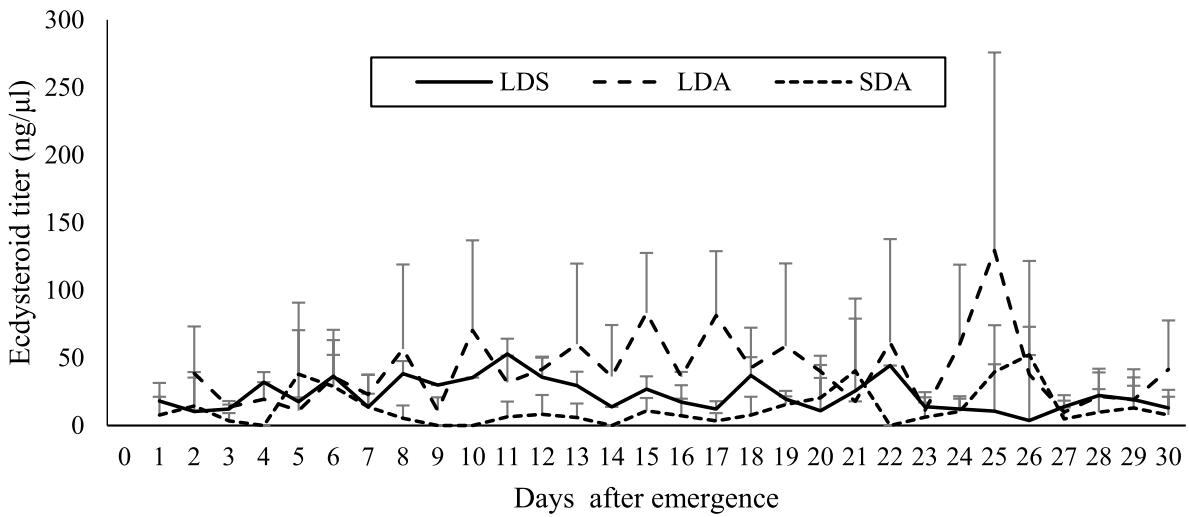




\section{Ovarian development}

Application of 5 or $50 \mu \mathrm{g}$ of methoprene significantly promoted ovarian development (methoprene $5 \mu \mathrm{g}$ vs control, $d f=1$, error $=0.02788, z=9.285, P<0.0001 ; 5 \mu \mathrm{g}$ methoprene vs no treatment, $d f=1$, error $=0.02696$, $z=-9.525, P<0.0001 ; 50 \mu \mathrm{g}$ methoprene vs control, $d f=1$, error $=0.032495, z=7.982, P<0.0001 ; 50 \mu \mathrm{g}$ methoprene vs no treatment, $d f=1$, error $=0.03171$, $z=-83.114, P<0.0001)$ on day 5 . Development varied strongly with dose, as $5 \mu \mathrm{g}$ of methoprene did not lead to development of mature eggs, while $50 \mu \mathrm{g}$ of methoprene stimulated egg maturation by day 5 (Table 1). By day 10 , both $5 \mu \mathrm{g}$ and $50 \mu \mathrm{g}$ of methoprene led to the presence of mature eggs. Neither the control nor the untreated group had developed ovaries by day 10 , indicating they were in a state of diapause.

Injection of $20 \mathrm{E}$ did not significantly promote ovarian development regardless of quantity $(1$ or $10 \mu \mathrm{g})$ in comparison with the control or the no-treatment groups on day $5(1 \mu \mathrm{g} 20 \mathrm{E}$ vs control, $d f=1$, error $=0.09344$, $z=1.227, P=0.734 ; 1 \mu \mathrm{g} 20 \mathrm{E}$ vs no treatment, $d f=1$, error $=0.010390, z=-0.775, P=0.937 ; 10 \mu \mathrm{g} 20 \mathrm{E}$ vs control, $d f=1$. error $=0.008679, z=-0.404, P=0.994$; $10 \mu \mathrm{g} 20 \mathrm{E}$ vs no treatment, $d f=1$, error $=0.009344$, $z=1.227, P=0.734)$ or $(1 \mu \mathrm{g} 20 \mathrm{E}$ vs control, $d f=1$, error $=0.02284, z=0.600, P=0.975 ; 1 \mu \mathrm{g} 20 \mathrm{E}$ vs no treatment, $d f=1$, error $=0.02086, z=-1.694$, $P=0.436 ; 10 \mu \mathrm{g} \mathrm{20E}$ vs control, $d f=1$. error $=0.02500$, $z=-0.866, P=0.909 ; 10 \mu \mathrm{g} 20 \mathrm{E}$ vs no treatment, $d f=1$, error $=0.0231, z=-1.864, P=0.335$ ) day 10 (Table 2). However, simultaneous application of methoprene $(5 \mu \mathrm{g})$ and $20 \mathrm{E}(1 \mu \mathrm{g})$ did significantly promote ovary development compared to the control or the notreatment group on day $5(5 \mu \mathrm{g}$ methoprene $+1 \mu \mathrm{g}$ $20 \mathrm{E}$ vs control, $d f=1$, error $=0.01172, z=10.245$, $P<0.0001 ; 5 \mu \mathrm{g}$ methoprene $+10 \mu \mathrm{g} 20 \mathrm{E}$ vs no treatment, $d f=1$. error $=0.01021, z=-9.303, P<0.0001$ )
Table 1 Effects of JHA (methoprene) on ovarian development of Polygonia c-aureum at 5 and 10 days post application
Table 2 Effects of 20-hydroxyecdysone on ovarian development of Polygonia c-aureum at 5 and 10 days post application

\begin{tabular}{llcl}
\hline Treatment & No. of insects & No. of eggs $(\text { mean } \pm \mathrm{SD})^{\mathrm{A}}$ & ${\text { Oocyte diameter }(\mathrm{mm})(\mathrm{mean} \pm \mathrm{SD})^{\mathrm{A}}}^{\text {On day } 5}$ \\
& 34 & $0.00 \pm 0.00^{\mathrm{a}}$ & $0.387 \pm 0.149^{\mathrm{a}}$ \\
JHA5 $\mu \mathrm{g}$ & 14 & $0.214 \pm 0.802^{\mathrm{b}}$ & $0.387 \pm 0.181^{\mathrm{a}}$ \\
JHA50 $\mu \mathrm{g}$ & 29 & $0.00 \pm 0.00^{\mathrm{a}}$ & $0.128 \pm 0.025^{\mathrm{b}}$ \\
Control & 29 & $0.130 \pm 0.030^{\mathrm{b}}$ \\
No treatment & 34 & $0.00 \pm 0.00^{\mathrm{a}}$ & \\
On day 10 & & & $0.387 \pm 0.149^{\mathrm{a}}$ \\
JHA5 $\mu \mathrm{g}$ & 34 & $50.480 \pm 35.548^{\mathrm{a}}$ & $0.699 \pm 0.063^{\mathrm{a}}$ \\
JHA50 $\mu \mathrm{g}$ & 29 & $68.931 \pm 39.864^{\mathrm{a}}$ & $0.159 \pm 0.038^{\mathrm{b}}$ \\
Control & 26 & $0.00 \pm 0.00^{\mathrm{b}}$ & $0.131 \pm 0.021^{\mathrm{b}}$ \\
No treatment & 30 & $0.00 \pm 0.00^{\mathrm{b}}$ & \\
\hline
\end{tabular}

${ }^{A}$ Means followed by different letters indicate a significant difference at the $5 \%$ level by Tukey method after ANOVA. Control is acetone

\begin{tabular}{lccc}
\hline Treatment & No. of insects & No. of eggs (mean \pm SD $)^{\mathrm{A}}$ & $\begin{array}{l}\text { Oocyte diameter }(\mathrm{mm}) \\
(\text { mean } \pm \mathrm{SD})^{\mathrm{A}}\end{array}$ \\
\hline On day 5 & & & \\
$20-\mathrm{OH} 1 \mu \mathrm{g}$ & 21 & $0.00 \pm 0.00^{\mathrm{a}}$ & $0.118 \pm 0.020^{\mathrm{a}}$ \\
$20-\mathrm{OH} 10 \mu \mathrm{g}$ & 27 & $0.00 \pm 0.00^{\mathrm{a}}$ & $0.133 \pm 0.023^{\mathrm{a}}$ \\
20-OH1 $\mu \mathrm{g}+\mathrm{JHA5} \mu \mathrm{g}$ & 16 & $0.00 \pm 0.00^{\mathrm{a}}$ & $0.225 \pm 0.067^{\mathrm{b}}$ \\
Control & 21 & $0.00 \pm 0.00^{\mathrm{a}}$ & $0.110 \pm 0.022^{\mathrm{a}}$ \\
No treatment & 34 & $0.00 \pm 0.00^{\mathrm{a}}$ & $0.130 \pm 0.030^{\mathrm{a}}$ \\
On day 10 & & & \\
20-OH1 $\mu \mathrm{g}$ & 26 & $0.00 \pm 0.00^{\mathrm{a}}$ & $0.166 \pm 0.061^{\mathrm{a}}$ \\
20-OH10 $\mu \mathrm{g}$ & 18 & $0.00 \pm 0.00^{\mathrm{a}}$ & $0.174 \pm 0.068^{\mathrm{a}}$ \\
20-OH1 $\mu \mathrm{g}+\mathrm{JHA5} \mu \mathrm{g}$ & 19 & $27.579 \pm 33.482^{\mathrm{b}}$ & $0.674 \pm 0.155^{\mathrm{b}}$ \\
Control & 21 & $0.00 \pm 0.00^{\mathrm{a}}$ & $0.153 \pm 0.047^{\mathrm{a}}$ \\
No treatment & 34 & $0.00 \pm 0.00^{\mathrm{a}}$ & $0.130 \pm 0.038^{\mathrm{a}}$ \\
\hline
\end{tabular}

A Means followed by different letters indicate a significant difference at the $5 \%$ level by Tukey method after ANOVA. Control is acetone 
and 10 (5 $\mu \mathrm{g}$ methoprene $+1 \mu \mathrm{g} 20 \mathrm{E}$ vs control, $d f=1$, error $=0.02465, z=21.155, P<0.0001 ; 5 \mu \mathrm{g}$ methoprene $+10 \mu \mathrm{g} 20 \mathrm{E}$ vs no treatment, $d f=1$. error $=0.02282, z=-23.793, P<0.0001$ ), further confirming that the promotion of ovarian development was due to application of methoprene and not $20 \mathrm{E}$.

\section{Testis development}

Treatment of autumn-form butterflies with either $5 \mu \mathrm{g}$ or $50 \mu \mathrm{g}$ of methoprene had no significant effect on testis development (5 $\mu \mathrm{g}$ methoprene vs control, $d f=1$, error $=0.10012, z=1.634, P=0.358 ; 50 \mu \mathrm{g}$ methoprene vs control, $d f=1$, error $=0.10172, z=-1.847, P=0.815$ ) 5 or $(50 \mu \mathrm{g}$ methoprene vs control, $d f=1$, error $=0.06910$, $z=-0.880, P=0.250 ; 50 \mu \mathrm{g}$ methoprene vs no treatment, $d f=1$, error $=0.06202, z=0.092, P=0.9997) 10$ days after treatment (at both time periods) (Table 3). At 10, 20, or 30 days after emergence, $\mathrm{JH}$ treatment groups, the control, and the untreated group, all showed a similar level of shrinkage of the testis (Fig. 3), indicating no effect of $\mathrm{JH}$ treatment on testes size. Similarly, treatment with $20 \mathrm{E}$ showed no effect on testes development, either 5 days $(20 \mathrm{E}$ $1 \mu \mathrm{g}$ vs control, $d f=1$, error $=0.10302, z=-2.191$, $P>0.180 ; 20 \mathrm{E} 10 \mu \mathrm{g} 1 \mathrm{l}$ v control, $d f=1$, error $=0.11213$, $z=-2.128, P>0.205)$ or 10 days (20E $1 \mu \mathrm{g}$ vs control, $d f=1$, error $=0.08267, z=0.200, P=0.9996 ; 20 \mathrm{E}$ $1 \mu \mathrm{g}$ vs no treatment, $d f=1$, error $=0.07387, z=1.227$, $P=0.734 ; 20 \mathrm{E} 10 \mu \mathrm{g}$ vs control, $d f=1$, error $=0.08404$, $z=-1.719, P=0.421$ ) after treatment (Table 4).

\section{Accessory gland development}

The accessory glands of butterflies treated with 5 or $50 \mu \mathrm{g}$ of methoprene were significantly heavier than the glands in the control or the no-treatment groups on day

Table 3 Effects of JHA methoprene on male reproductive development of Polygonia c-aureum on days 5 and 10 after treatment

\begin{tabular}{lllll}
\hline Treatment & No. of insects & Testis size $(\text { mean } \pm \mathrm{SD})^{\mathrm{A}}$ & Mass of accessory glands $(\mathrm{mg})(\mathrm{mean} \pm \mathrm{SD})^{\mathrm{A}}$ & Mass of simplex $(\mathrm{mg})(\mathrm{mean} \pm \mathrm{SD})^{\mathrm{A}}$ \\
\hline On day 5 & & & & $1.800 \pm 0.458^{\mathrm{ab}}$ \\
JHA5 $\mu \mathrm{g}$ & 31 & $1.481 \pm 0.348^{\mathrm{a}}$ & $0.217 \pm 0.087^{\mathrm{b}}$ & $1.988 \pm 0.413^{\mathrm{b}}$ \\
JHA50 $\mu \mathrm{g}$ & 17 & $1.456 \pm 0.338^{\mathrm{a}}$ & $0.368 \pm 0.113^{\mathrm{ab}}$ & $1.589 \pm 0.319^{\mathrm{a}}$ \\
Control & 22 & $1.540 \pm 0.336^{\mathrm{a}}$ & $0.116 \pm 0.069^{\mathrm{b}}$ & $1.589 \pm 0.346^{\mathrm{a}}$ \\
No treatment & 18 & $1.644 \pm 0.223^{\mathrm{a}}$ & $0.142 \pm 0.096^{\mathrm{b}}$ & $2.742 \pm 0.748^{\mathrm{a}}$ \\
& & & & $2.753 \pm 0.651^{\mathrm{a}}$ \\
On day 10 & & & & $2.048 \pm 0.467^{\mathrm{b}}$ \\
JHA5 $\mu \mathrm{g}$ & 26 & $0.880 \pm 0.236^{\mathrm{a}}$ & $0.369 \pm 0.113^{\mathrm{a}}$ & $1.836 \pm 0.400^{\mathrm{b}}$ \\
JHA50 $\mu \mathrm{g}$ & 33 & $1.152 \pm 0.277^{\mathrm{ab}}$ & $0.383 \pm 0.138^{\mathrm{a}}$ & $0.160 \pm 0.090^{\mathrm{b}}$ \\
Control & 25 & $1.213 \pm 0.175^{\mathrm{b}}$ & $0.116 \pm 0.069^{\mathrm{b}}$ & \\
No treatment & 38 & $1.158 \pm 0.306^{\mathrm{ab}}$ & 0.116 & \\
\hline
\end{tabular}

A Means followed by different letters indicate a significant difference at the $5 \%$ level by Tukey method after ANOVA. Control is acetone

Fig. 3 Effects of methoprene (JHA) on the testis size in adults of various ages in Polygonia c-aureum. Different letters indicate significant differences

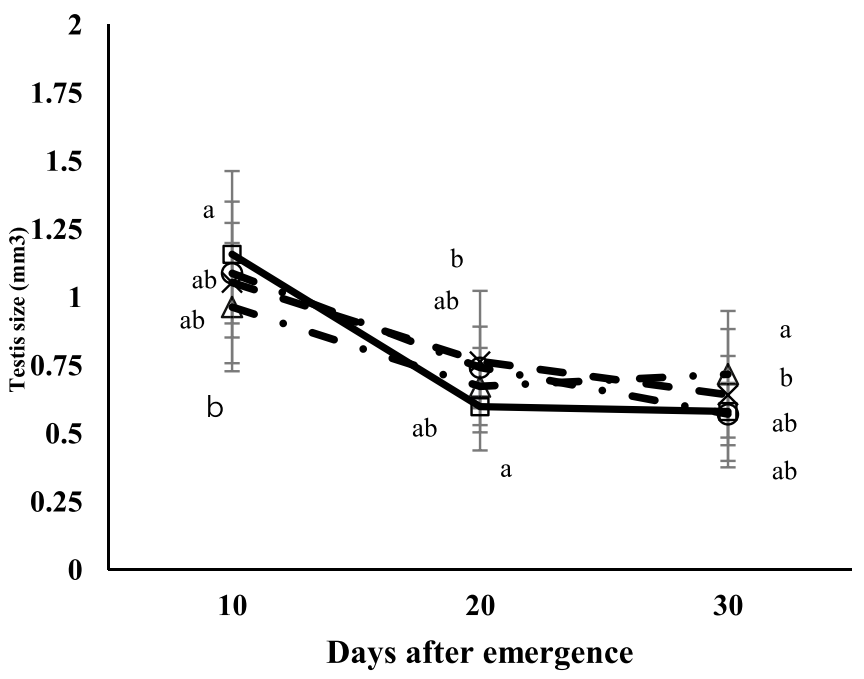


Table 4 Effects of 20-hydroxyecdysone on male reproductive development of Polygonia c-aureum on days 5 and 10 after treatment

\begin{tabular}{|c|c|c|c|c|c|}
\hline \multirow{2}{*}{$\begin{array}{l}\text { Treatment } \\
\text { On day } 5\end{array}$} & \multicolumn{2}{|c|}{ No. of insects } & \multirow[t]{2}{*}{$\begin{array}{l}\text { Testis size }\left(\mathrm{mm}^{3}\right) \\
(\text { mean } \pm \mathrm{SD})^{\mathrm{A}}\end{array}$} & \multirow[t]{2}{*}{$\begin{array}{l}\text { Mass of accessory glands }(\mathrm{mg}) \\
(\mathrm{mean} \pm \mathrm{SD})^{\mathrm{A}}\end{array}$} & \multirow[t]{2}{*}{$\begin{array}{l}\text { Mass of simplex }(\mathrm{mg}) \\
(\text { mean } \pm \mathrm{SD})^{\mathrm{A}}\end{array}$} \\
\hline & & & & & \\
\hline $20 \mathrm{E} 1 \mu \mathrm{g}$ & 34 & $1.576 \pm$ & & $0.090 \pm 0.078^{\mathrm{a}}$ & $1.640 \pm 0.425^{\mathrm{a}}$ \\
\hline $20 \mathrm{E} 10 \mu \mathrm{g}$ & 23 & $1.589 \pm$ & & $0.180 \pm 0.096^{\mathrm{b}}$ & $1.887 \pm 0.38^{\mathrm{ab}}$ \\
\hline $20 \mathrm{E} 1 \mu \mathrm{g}+\mathrm{JHA} 5 \mu \mathrm{g}$ & 18 & $1.422 \pm$ & & $0.266 \pm 0.101^{\mathrm{a}}$ & $2.111 \pm 0.164^{\mathrm{b}}$ \\
\hline Control & 16 & $1.36 \pm$ & & $0.120 \pm 0.103^{\mathrm{ab}}$ & $1.660 \pm 0.232^{\mathrm{a}}$ \\
\hline No treatment & 34 & $1.540 \pm$ & & $0.116 \pm 0.069^{\mathrm{a}}$ & $1.589 \pm 0.318^{\mathrm{a}}$ \\
\hline \multicolumn{6}{|l|}{ On day 10} \\
\hline $20 \mathrm{E} 1 \mu \mathrm{g}$ & 23 & $1.198 \pm$ & & $0.209 \pm 0.092$ & $1.887 \pm 0.387^{\mathrm{a}}$ \\
\hline $20 \mathrm{E} 10 \mu \mathrm{g}$ & 27 & $1.368 \pm$ & & $0.209 \pm 0.00$ & $2.016 \pm 0.438^{\mathrm{a}}$ \\
\hline $20 \mathrm{E} 1 \mu \mathrm{g}+\mathrm{JHA} 5 \mu \mathrm{g}$ & 22 & $1.188 \pm$ & & $0.400 \pm 0.157^{\mathrm{a}}$ & $2.880 \pm 0.547^{b}$ \\
\hline Control & 24 & $1.214 \pm$ & & $0.116 \pm 0.088$ & $1.877 \pm 0.412^{\mathrm{a}}$ \\
\hline No treatment & 35 & $1.158 \pm$ & & $0.142 \pm 0.096$ & $1.836 \pm 0.400^{\mathrm{a}}$ \\
\hline
\end{tabular}

${ }^{\text {A }}$ Means followed by different letters indicate a significant difference at the $5 \%$ level by Tukey method after ANOVA. Control is acetone

$5(d f=1$, error $=0.02781, z$ value $=3.297, P<0.01$; $d f=1$, error $=0.02578, z$ value $=-3.920, P<0.001$, respectively) or day 5 ( $5 \mu \mathrm{g}$ methoprene vs control, $d f=1$, error $=0.03177, z=6.587, P<0.0001 ; 5 \mu \mathrm{g}$ methoprene vs no treatment, $d f=1$, error $=0.02919, z=-7.797$, $P<0.0001 ; 50 \mu \mathrm{g}$ methoprene vs control, $d f=1$, error $=0.02970, z=7.504, P<0.0001 ; 50 \mu$ g methoprene vs no treatment, $d f=1$, error $=0.02692, z=-8.959$, $P<0.0001$ (Table 3). At 10, 20, or 30 days after emergence, males in both methoprene treatment groups showed greater development of the accessory gland than males in the control or untreated groups, especially on day 30 (5 $\mu \mathrm{g}$ methoprene vs control, $d f=1$, error $=0.06202$, $z=6.046, P<0.001 ; 50 \mu \mathrm{g}$ methoprene vs control, $d f=1$, error $=0.05759, v=6.512, P<0.001)($ Fig. 4$)$.
Injection of autumn-form butterflies with $20 \mathrm{E}$ 2 days after emergence had no effect on accessory gland development by day $5(1 \mu \mathrm{g} 20 \mathrm{E}$ vs control, $d f=1$, error $=0.02683, z=1.118, P=0.795 ; 10 \mu \mathrm{g}$ $20 \mathrm{E}$ vs control, $d f=1$, error $=0.02885, v=-2.095$, $P=0.2)$ or $10(1 \mu \mathrm{g} 20 \mathrm{E}$ vs no treatment, $d f=1$, error $=0.02683, \mathrm{z}=-2.519, P=0.0859 ; 10 \mu \mathrm{g} 20 \mathrm{E}$ vs control, $d f=1$, error $=0.02957, z=-3.202, P<0.012$ ) (Table 4). In contrast, the simultaneous application of both $20 \mathrm{E}$ and methoprene did significantly affect accessary gland development on days 5 ( $5 \mu \mathrm{g}$ methoprene $+1 \mu \mathrm{g} 20 \mathrm{E}$ vs control, $d f=1$, error $=0.03000$, $z=4.856, P<0.001)$ and $10(5 \mu \mathrm{g}$ methoprene $+1 \mu \mathrm{g}$ $20 \mathrm{E}$ vs control, $d f=1$,error $=0.03111, z=9.174$, $P<0.001$ ) (Table 4).
Fig. 4 Effects of methoprene (JHA) on the development of male accessory glands in adults of various ages in Polygonia c-aureum. Different letters indicate significant differences

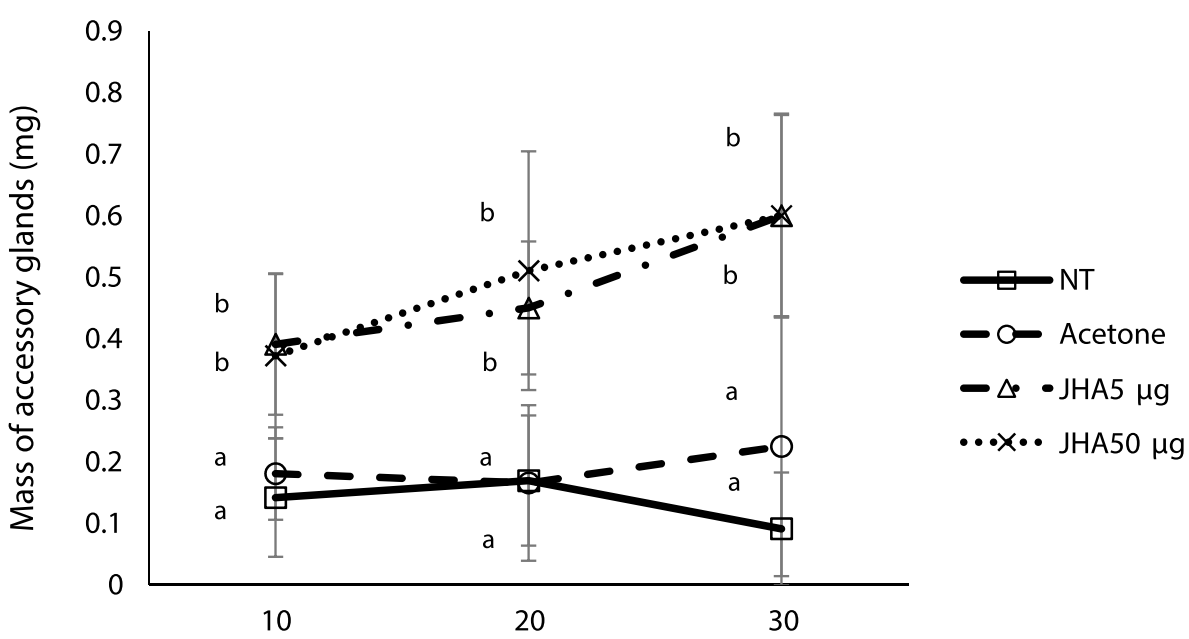

Days after emergence 


\section{Simplex development}

To examine the effect of the application of methoprene on simplex development, autumn-form butterflies were treated with 5 or $50 \mu \mathrm{g}$ of methoprene, acetone, or were left completely untreated and were examined on days 5 and 10 (Table 3). The mass of the simplex of the $50 \mu \mathrm{g}$ methoprene treatment was significantly heavier than that of the controls or individuals in the no-treatment group on day $5(d f=1$, error $=0.12111, z=3.299, P<0.01 ; d f=1$, error $=0.11256, z=-3.546, P<0.01$, respectively). On day 10 , there were significant differences between either the groups treated with 5 or $50 \mu \mathrm{g}$ of methoprene, compared with either the control or the untreated group $(5 \mu \mathrm{g}$ methoprene vs control, $d f=1$, error $=0.06202, z=6.046$, $P<0.001 ; 5 \mu \mathrm{g}$ methoprene vs no treatment, $d f=1$, error $=0.1500, z=-6.043, P<0.0001 ; 50 \mu \mathrm{g}$ methoprene vs control, $d f=1$, error $=0.1500, z=4.698, P<0.0001$; $50 \mu \mathrm{g}$ methoprene vs control, $d f=1$, error $=0.1366$, $z=-6.712, P<0.0001)$. To examine the effect of the methoprene treatment over the long term on simplex development, animals were examined 10, 20, or 30 days after emergence (Fig. 5) and we found significant differences between methoprene-treated groups and either the control or the untreated group at all ages, especially on day 30 (5 $\mu \mathrm{g}$ methoprene vs control, $d f=1$, error $=0.06202$, $z=6.046, P<0.001 ; 5 \mu \mathrm{g}$ methoprene vs no treatment, $d f=1$, error $=0.1500, z=-6.043, P<0.0001 ; 50 \mu \mathrm{g}$ methoprene vs control, $d f=1$, error $=0.1500, z=4.698$, $P<0.0001 ; 50 \mu \mathrm{g}$ methoprene vs control, $d f=1$, error $=0.1366, z=-6.712, P<0.0001)$. There were, however, no significant differences among $20 \mathrm{E}$ treatments on day 5 ( $1 \mu \mathrm{g} 20 \mathrm{E}$ vs control, $d f=1$, error $=0.11775$, $z=-0.677, P=0.9609 ; 1 \mu \mathrm{g} 20 \mathrm{E}$ vs no treatment, $d f=1$, error $=0.09357, z=-0.541, P=0.9827 ; 10 \mu \mathrm{g} 20 \mathrm{E}$ vs control, $d f=1$, error $=0.12608, z=-2.438, P=0.1039$; $10 \mu \mathrm{g} 20 \mathrm{E}$ vs no treatment, $d f=1$, error $=0.10385$, $z=-2.680, P=0.0557)$ and day 10 (1 $\mu \mathrm{g} 20 \mathrm{E}$ vs control, $d f=1$, error $=0.1246, z=-1.105, P=0.803 ; 1 \mu \mathrm{g}$ $20 \mathrm{E}$ vs no treatment, $d f=1$, error $=0.1138, z=-1.574$, $P=0.513 ; 10 \mu \mathrm{g} 20 \mathrm{E}$ vs control, $d f=1$, error $=0.1246$, $v=-1.105, P=0.80$ ) (Table 4), except for between the simultaneous application of methoprene and $20 \mathrm{E}$ on days 5 and 10 (5 $\mu \mathrm{g}$ methoprene $+1 \mu \mathrm{g} 20 \mathrm{E}$ vs control, $d f=1$, error $=0.13121, z=4.196, P<0.001 ; 0.5 \mu \mathrm{g}$ methoprene $+1 \mu \mathrm{g} 20 \mathrm{E}$ vs control, $d f=1$, error $=0.1311$, $z=8.410, P<0.0001$, respectively).

\section{Sperm movement}

Application of $5 \mu \mathrm{g}$ of methoprene significantly promoted eupryrene sperm movement in comparison with the control group $(d f=1$, error $=19.803, z=0.0298$, $P=0.030)$ on day 10 , but this was not the case with the untreated group $(d f=1$, error $=19.803, z=-0.747$, $P=0.8778$ ) (Table 5). Similar results on application of $20 \mathrm{E}$ were obtained on day 10 ( $1 \mu \mathrm{g} 20 \mathrm{E}$ vs control, $d f=1$, error $=0.1819, z=-3.623, P=0.030 ; 1 \mu \mathrm{g}$ $20 \mathrm{E}$ vs no treatment, $d f=1$, error $=0.1819, z=-1.687$, $P=0.442 ; 10 \mu \mathrm{g} 20 \mathrm{E}$ vs control, $d f=1$, error $=0.1819$, $z=-3.623, P=0.0026 ; 10 \mu \mathrm{g} 20 \mathrm{E}$ vs no treatment, $d f=1$, error $=0.1819, z=-1.687, P=0.442)$ (Table 6). Simultaneous application of methoprene and $20 \mathrm{E}$ did not affect sperm movement.
Fig. 5 Effects of methoprene (JHA) on the development of simplex in adults of various ages in Polygonia c-aureum. Different letters indicate significant differences

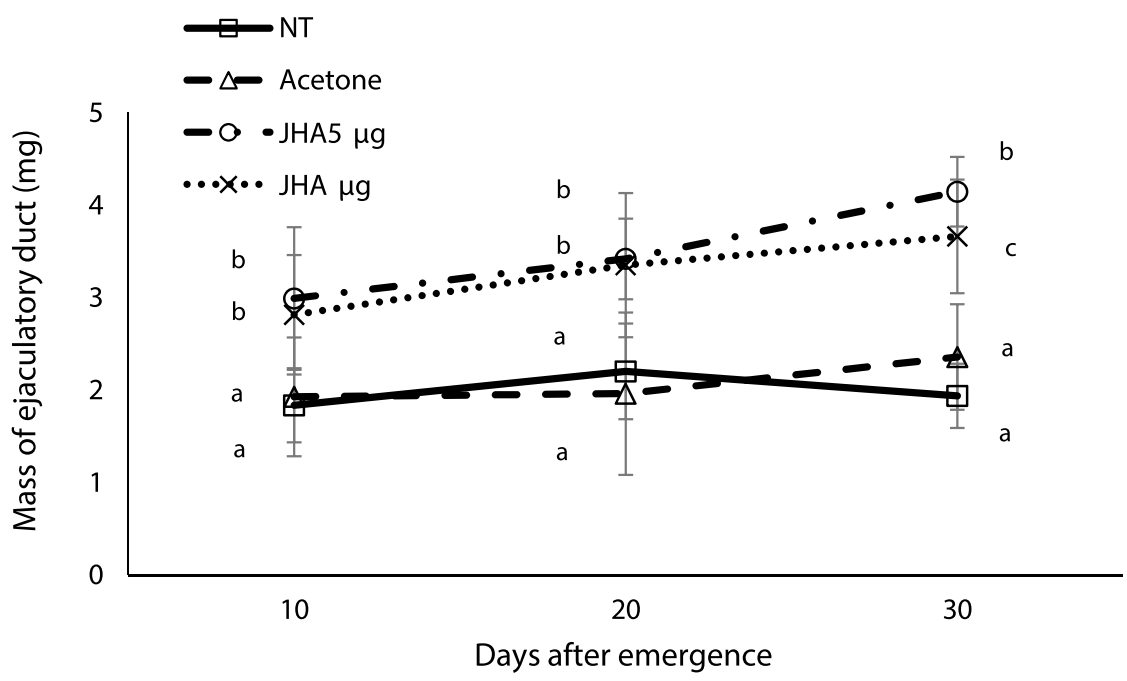


Table 5 Effects of JHA methoprene on sperm movement of Polygonia c-aureum on day 10 after treatment

\begin{tabular}{lll}
\hline Treatment & No. of insects & $\begin{array}{l}\text { No. of ESB in the duplex } \\
(\text { average } \pm \text { SD) }\end{array}$ \\
\hline JHA5 $\mu \mathrm{g}$ & 10 & $228.0 \pm 44.880^{\mathrm{a}}$ \\
JHA50 $\mu \mathrm{g}$ & 12 & $205.9 \pm 55.669^{\mathrm{ab}}$ \\
Control & 10 & $173.5 \pm 25.774^{\mathrm{b}}$ \\
No treatment & 10 & $213.2 \pm 42.570^{\mathrm{ab}}$ \\
\hline
\end{tabular}

A Means followed by different letters indicate a significant difference at the $5 \%$ level by Tukey method after ANOVA. Control is acetone

\section{Discussion}

Studies in many insect species have shown that the induction and maintenance of adult diapause in males and females is due to the inactivity of hormone production in the brain and CA, e.g., in Pyrrhocoris apterus (L.) (Hodková 1977a), in the blow fly Protophormia terraenovae Robineau-Desvoidy (Shiga et al. 2003), and in various other insects (Denlinger 2002). It is known that transplantation or allatectomy of CA and application of JHA can induce copulative behavior and development of the reproductive organs. For example, application of JH or JHA onto diapausing adults induced yolk deposition in the soybean pest Riptortus clavatus Thunberg (Numata and Hidaka 1984), mating behavior in the Caribbean fruit fly A. suspensa (Loew) (Teal et al. 2000), and calling behavior in the tephritid fly A. obliqua (Macquart) (Chacón-Benavente et al. 2013). In Lepidoptera, several studies have examined the effects of hormones on male reproductive development in monarch butterflies, Danaus plexippus (L.) (Herman 1973, 1975a, b; Barker and Herman 1976; Herman and Barker 1976; Herman 1981; Herman et al. 1981). These studies found that the application of $\mathrm{JH}$ and ecdysteroid promotes male accessory gland and tubular gland (=simplex) development in monarchs, and the simultaneous application of these hormones synergistically promotes this development. Moreover, Lessman et al. (1989) reported JH titer in a diapausing generation of $D$. plexippus to be lower than that in a non-diapausing generation. In $P$. c-aureum in this study, the effects of JHA on male reproductive development were similar to those seen previously in monarchs. However, 20-hydroxyecdysone did not affect the development of accessory gland and the simplex in males, unlike methoprene, which promoted it (Figs. 4, 5). These results indicate, for the first time for an adult diapausing lepidopteran, that 20-hydroxyecdysone did not affect reproduction of either sex of a butterfly. This difference seems to be due to differences between our study species and monarch butterflies, although both belong to the same family, the Nymphalidae.

No effects of ecdysteroid on male reproductive organ development were observed, nor were there synergistic effects of JHA and 20E (Tables 3, 4). Similar results were obtained for the milkweed bug Oncopeltus fasciatus (Dallas) by Bryan et al. (1974). In this study, we found the development of accessory gland and simplex was not different between adults treated with $20 \mathrm{E}$ and the control or untreated groups (Table 4). This suggests the possibility that any enhancement of the development of these organs would be limited to the time near adult eclosion in this butterfly, because the male's reproductive organs, except the testis, begin to enlarge abruptly during toward the end of the pupal stage and the first days of the adult stage (Hiroyoshi, unpublished data). As demonstrated in our study (Figs. 1, 2), ecdysteroid titer is generally low in adult lepidopterans (Bollenbacher et al. 1981; Kawasaki et al. 1986). Although we did not measure the ecdysteroid titer within the testis, it has been demonstrated that in lepidopterans the testis can synthesize ecdysteroids when stimulated by ecdysiotropin (Loeb et al. 1982, 1984, 1988, 2001). Thus, the effects of the ecdysteroid titer of the testis on male reproduction should be examined in future studies.

In $P$. c-aureum, the testis starts to shrink at the end of the pupal stage, and this continues throughout the adult stage and appears to be common in lepidopteran species (Hiroyoshi 2000). In the present study, an application of methoprene caused a complicated response (Table 3), while application of 20E did not affect testis size (Table 4). Since diapause does not affect testis shrinkage (Hiroyoshi 2000),
Table 6 Effects of

20-hydroxyecdysone on sperm movement of Polygonia c-aureum on day 10 after treatment

\begin{tabular}{lll}
\hline Treatment & No. of insects & No. of ESB in the duplex (average \pm SD) \\
\hline $20 \mathrm{CH} 1 \mu \mathrm{g}$ & 10 & $204.7 \pm 21.702^{\mathrm{a}}$ \\
$20 \mathrm{CH} 10 \mu \mathrm{g}$ & 9 & $243.9 \pm 31.947^{\mathrm{b}}$ \\
$20 \mathrm{CH} 1 \mu \mathrm{g}+\mathrm{JHA} 5 \mu \mathrm{g}$ & 10 & $204.2 \pm 43.228^{\mathrm{ab}}$ \\
Control & 10 & $173.5 \pm 25.774^{\mathrm{b}}$ \\
No treatment & 10 & $213.2 \pm 42.570^{\mathrm{ab}}$ \\
\hline
\end{tabular}

${ }^{A}$ Means followed by different letters indicate a significant difference at the $5 \%$ level byTukey method after ANOVA. Control is acetone 
we conclude that neither of these two hormones affects testis shrinkage. However, we cannot exclude the possibility that a low titer of ecdysteroids might promote testis shrinkage by itself.

In the spotted stalk borer, Chilo partellus (Swinhoe), uptake of larval protein by the accessory glands in 8- to 10 -day-old adults was promoted by $20 \mathrm{E}$ in vitro (Bajaj et al. 1990; Ismail et al. 1993). Although ecdysteroids stimulated cell division and imaginal differentiation of accessory glands or synthesis of proteins during the pupal stage in the mealworm beetle Tenebrio molitor L. (Szopa et al. 1985; Grimnes and Happ 1987; Happ 1987; Yaginuma et al. 1988; Sridevi et al. 1988), the receptivity or function of hormone changes depends on the stage. For example, in T. molitor, it has been suggested that commitment to the production of trehalase in adult accessory glands occurs during the pupal stage (Yaginuma and Happ 1989). Thus, the accessory glands may have a sensitivity for ecdysteroids around the time of adult eclosion in P. c-aureum. Although the application of 20E onto 2-day-old adults did not promote the development of accessory gland and simplex in P. c-aureum (Table 4), it is possible that this stage of application might be too late. Ecdysteroid titers were low in both sexes of P. c-aureum in the adult stage (Figs. 1, 2). We had previously measured ecdysteroid titer in individuals every $3 \mathrm{~h}$ from $1 \mathrm{~h}$ after initiation of light to $24 \mathrm{~h}$ (data not shown) and found no differences in titer among times. This suggests that the low ecdysteroid titer seen in the above experiments (Figs. 1, 2) was not due to the sampling time, although in another study, Galleria mellonella (L.) larvae exhibited a circadian rhythm of ecdysteroids titer (Cymborowski et al. 1989).

$\mathrm{JH}$ is necessary to stimulate vitellogenin synthesis in the boll weevil, Anthonomus grandis Boheman (TaubMontemayor and Rankin 1997). In Lepidoptera, it was first reported that $\mathrm{JH}$ induced vitellogenin synthesis in D. plexippus (Pan and Wyatt 1971). Thereafter, it was shown that JH breaks ovarian diapause in the nymphalids Inachis io (L.) and Aglais urticae (L.) (Benz 1972). Ecdysone can regulate vitellogenesis in Diptera, since it affects rates of vitellogenin synthesis in females under various experimental regimes (Bownes 1989). JH synthesized de novo in CA stimulates ecdysone biosynthesis by the ovarian follicular cells (Gruntenko et al. 2012), and $\mathrm{JH}$ and ecdysteroids act similarly on the yolk protein metabolism of Apis mellifera L. (Wegener et al. 2013). Gruntenko and Rauschenbach (2008) suggest that ecdysteroids make a bigger contribution to the control of early stages of vitellogenesis under stress conditions (heat shock), while JH is more important to the completion of egg maturation in D. melanogaster. Moreover, simultaneous application of methoprene and $20 \mathrm{E}$ was found to protect early vitellogenic oocytes from 20E-induced resorption in D. melanogaster (Soller et al. 1999). Recent studies have further clarified that a daily injection of neuropeptide F (NPF) into female adults of Schistocerca gregaria elicits an increase of oocyte size (van Wielendaele et al. 2013). In the present study, $20 \mathrm{E}$ did not affect ovarian development in adults (Table 2), although methoprene did promote ovarian development (Tables 1, 2). However, we cannot exclude the possibility that $20 \mathrm{E}$ promotes ovarian development in the pupal stage. As the effect of ecdysteroids on ovarian development in adult Lepidoptera is less understood, further research is needed to clarify the function of ecdysteroids in female reproduction.

$\mathrm{JH}$ promotes the development and function of male accessory glands in various insects (Chen 1984; Happ 1992; Gillott 1996). The development of accessory glands, including the synthesis of proteins by $\mathrm{JH}$ has been found in male adults of several species, including the mosquito A. aegypti (Ramalingam and Craig 1977), the grasshopper Melanoplus sanguinipes (Fabricius) (Venkatesh and Gillott 1983), the kissing bug Rhodnius prolixus Stål (Barker and Davey 1983), the black cutworm Agrotis ipsilon (Hufnagel) (Duportets et al. 1998), and the red flour beetle Tribolium castaneum (Herbst) (Parthasarathy et al. 2009). In the leafmining moth Caloptiiia fraxineiia (Ely), treatment with methoprene in autumn tended to increase the total protein concentration when compared with that of untreated control moths (Lemmen et al. 2016). JH deficiency has been shown to lower protein synthesis in the male accessory gland of D. melanogaster (Wilson et al. 2003). In the South American fruit fly Anastrepha fraterculus Wiedemann, inhibition of sexual receptivity of mated females is mediated by products in male accessory glands (Abraham et al. 2012). In P. c-aureum, development of MAG and simplex was promoted by methoprene (Figs. 4, 5; Table 3). This would be related to suppression of mating in a diapausing generation of this butterfly, because spermatophores mainly consist of substances from these organs.

Sperm movement of $P$. c-aureum was promoted by JHA (Table 5) and 20E (Table 6), although there were no significant differences between treated adults and those in the control or the untreated group. Although factors regulating sperm movement in lepidopteran insects are not fully understood, $\mathrm{JH}$ in hemolymph seems not to be involved in sperm movement, at least in adults, because sperm movement in P. c-aureum was independent of diapause (Hiroyoshi and Mitsuhashi 1998). On the other hand, ecdysteroids might be involved in sperm movement. In fact, Seth et al. (2004) demonstrated that the application of sublethal concentrations of the bisachylhydrazine molting hormone agonists RH-5849 and tebufenozide (RH-5992) led to dosedependent reductions in sperm movement in the Oriental leafworm moth, Spodoptera litura. In P. c-aureum, apyrene sperm movement starts at the end of the pupal stage, 
while eupyrene sperm movement starts around adult eclosion (Hiroyoshi 1997). It has been suggested that the commencement of sperm movement at the end of the pupal stage is related to a decline in ecdysteroid titer (Shimizu 1989). Thus, a low ecdysteroid titer in the hemolymph and/ or testis would promote sperm movement in adults of lepidopteran insects. However, the role of JH in sperm movement requires further research.

Acknowledgements We would like to thank Dr. N. Agui, Dr. T. Shimizu and Mr. M. Wani for their help. Thanks are also due to Dr. S. Moriya, Dr. K. Tateishi, and Mr. K. Takasino for sending the materials.

\section{Compliance with ethical standards}

Human and animal rights All applicable international, national, and/ or institutional guidelines for the care and use of animals were followed. All procedures performed in studies involving animals were in accordance with the ethical standards of the institution or practice at which the studies were conducted.

Ethical standards We have not submitted our manuscript to other journal at present.

Conflict of interest No competing interests declared.

\section{References}

Abraham S, Cladera J, Goane L, Vera MT (2012) Factors affecting Anastrepha fraterculus female receptivity modulation by accessory gland products. J Insect Physiol 58:1-6

Abraham S, Nuñez-Beverido N, Contreras-Navarro Y, Pérez-Staples D (2014) Female receptivity in Anastrepha ludens (Diptera: Tephritidae) is not modulated by male accessory gland products. J Insect Physiol 70:41-48

Bajaj JP, Ismail SM, Dutta-Guputa (Ray) A (1990) Uptake of Corcyra larval heamolymph proteins by the male accessory reproductive glands of stem borer, Chilo partellus (Lepidoptera-Insecta). Biochem Inter 20:549-554

Barker JF, Davey KG (1983) A polypeptide from the brain and corpus cardiacum of male Rhodnius prolixus which stimulates in vitro protein synthesis in the transparent accessory reproductive gland. Insect Biochem 13:7-10

Barker JH, Herman WS (1976) Effect of photoperiod and temperature on reproduction of the monarch butterfly, Danaus plexippus. J Insect Physiol 22:1565-1568

Benz G (1972) Juvenile hormone breaks ovarian diapause in two nymphalidae butterflies. Experientia 28:1507

Bollenbacher WE, Smith SL, Goodman W, Gilbert LI (1981) Ecdysteroid titer during larval-pupal-adult development of the tobacco hornworm, Manduca sexta. Gen Comp Endocrin 44:302-306

Borovsky D, Carlson DA, Hancock RG, Rembold H, Van Handel E (1994) De novo biosynthesis of juvenile hormone III and I by the accessory glands of the male mosquito. Insect Biochemi Mol Biol 24:437-444

Bownes M (1989) The roles of juvenile hormone, ecdysone and the ovary in the control of Drosophila vitellogenesis. J Insect Physiol 35:409-413
Briers T, De Loof A (1981) Moulting hormone activity in the adult Colorado potato beetle, Leptinotarsa decemlineata $\mathrm{S}_{\mathrm{AY}}$ in relation to reproduction and diapause. Int J Invertebr Reprod Dev 3:145-155

Briers T, Peferoen M, De Loof A (1982) Ecdysteroids and adult diapause in the Colorado potato beetle, Leptiotarsa decemlineata. Physiol Entomol 7:379-386

Bryan MD, Brown TM, Monroe RE (1974) Effect of ecdysterone on ethyl trimethl dodecadienoate juvenile hormone action in Oncopeltus fasciatus. J Insect Physiol 20:1057-1061

Chacón-Benavente R, López-Guillen G, Hernández E, Rojas JC, Malo EA (2013) Juvenile hormone analog enhances calling behavior, mating success, and quantity of volatiles released by Anastrepha obliqua (Diptera: Tephritidae). Environ Entomol 42:262-269

Chen PS (1984) The functional morphology and biochemistry of insect male accessory glands and their secretions. Ann Rev Entomol 29:233-255

Clifton ME, Corre S, Rivera-Perez C, Nouzova M, Noriega FG (2014) Male Aedes aegypti mosquitoes use JH III transferred during copulation to influence previtellogenic ovary physiology and affect the reproductive output of female mosquitoes. J Insect Physiol 64:40-47

Cymborowski B, Śmietanko A, Delbecque JP (1989) Circadian modulation of ecdysteroid titer in Galleria mellonella larvae. Comp Biochem Physiol 94A:431-438

Davey KG (2000) The modes of action of juvenile hormones: some questions we ought to ask. Insect Biochem Mol Biol 30:663-669

De Kort CAD, Khan MA, Koopmanschap AB (1987) Juvenile hormone and the control of adult diapause in the Colorado potato beetle, Leptinotarsa decemlineata. Insect Biochem 17:985-988

Denlinger DL (2002) Regulation of diapause. Ann Rev Entomol 47:93-122

Dinan L (1997) Ecdysteroids in adults and eggs of the house cricket, Acheta domesticus (Orthoptera: Gryllidae). Comp Biochem Physiol 116B:129-135

Dottorini T, Nicolaides L, Ranson H, Rogers DW, Crisanti A, Catteruccia F (2007) A genome-wide analysis in Anopheles gambiae mosquitoes reveals 46 male accessory gland genes, possible modulators of female behavior. PNAS 104:162158

Duportets L, Dufour MC, Couillaud F, Gadenne C (1998) Biosynthetic activity of corpora allata, growth of sex accessory glands and mating in the male moth Agrotis ipsilon (Hufnagel). J Exp Biol 201:2425-2432

Endo K (1973a) Hormonal regulation of mating in the butterfly, Polygonia c-aureum L. Dev Growth Differ 15:1-10

Endo K (1973b) Relation between accumulation of secretory fluid in the accessory gland of the female genital organ and activity of the corpus allatum in Polygonia c-aureum. Zool Mag 82:53-58

Ganter GK, Panaitiu AE, Desilets JB, Davis-Helm JA, Fisher EA, Tan LCH, Heinrich R, Buchanan EB, Brooks KM, Kenney MT, Verde MG, Downey J, Adams AM, Grenier JS, Maddula S, Shah P, Kincaid KM, O'Brien JRM (2011) Drosophila male courtship behavior is modulated by ecdysteroids. J Insect Physiol 57:1179-1184

Gilbert LI, Granger NA, Roe RM (2000) The juvenile hormones: historical facts and speculations on future research directions. Insect Biochem Mol Biol 30:617-644

Gillott C (1996) Male insect accessory glands: Functions and control of secretory activity. Invertebr Reprod Dev 30:199-205

Gillott C (2003) Male accessory gland secretion: modulators of female reproductive physiology and behavior. Ann Rev Entomol 48:163-184

Gillott C, Ismail PM (1995) In vitro synthesis of ecdysteroid by the male accessory reproductive glands, testis and abdominal 
integument of the adult migratory grasshopper, Melanoplus sanguinipes. Invertebr Reprod Dev 27:65-71

Grimnes KA, Happ GM (1987) Ecdysteroids in vitro promote differentiation in the accessory glands of male mealworm beetles. Experientia 43:906-907

Gruntenko NE, Rauschenbach IY (2008) Interplay of JH, 20E and biogenic amines under normal and stress conditions and its effect on reproduction. J Insect Physiol 54:902-908

Gruntenko NE, Laukhina OV, Yu Rauschenbach I (2012) Role of D1- and D2-like receptors in age-specific regulation of juvenile hormone and 20-hydroxyecdysone levels by dopamine in Drosophila. J Insect Physiol 58:1534-1540

Happ GM (1987) Accessory gland development in mealworm beetles. In: Alan R (ed) Molecular Entomology. Liss. Inc, New York, pp 433-434

Happ GM (1992) Maturation of the male reproductive system and its endocrine regulation. Ann Rev Entomol 37:303-320

Hassanien ITE, Grötzner M, Meyering-Vos M, Hoffmann KH (2014) Neuropeptides affecting the transfer of juvenile hormones from males to females during mating in Spodoptera frugiperda. J Insect Physiol 66:45-52

Herman WS (1973) The endocrine basis of reproductive inactivity in monarch butterflies overwintering in central California. J Insect Physiol 19:1883-1887

Herman WS (1975a) Juvenile hormone stimulation of tubular and accessory glands in male monarch butterflies. Comp Biochem Physiol 51A:507-510

Herman WS (1975b) Endocrine regulation of posteclosion enlargement of the male and female reproductive glands in monarch butterflies. Gend Comp Endocrin 26:534-540

Herman WS (1981) Studies on the adult reproductive diapause o f the monarch butterfly, Danaus plexippus. Biol Bull 160:89-106

Herman WS, Barker JF (1976) Ecdysterone antagonism, mimicry, and synergism of juvenile hormone action on the monarch butterfly reproductive tract. J Insect Physiol 22:643-648

Herman WS, Lessman CA, Johnson GD (1981) Correlation of juvenile hormone titer changes with reproductive tract development in the post eclosion monarch butterfly. J Exp Zool 218:387-395

Hidaka T, Takahashi H (1967) Temperature condition s and maternal effect as modifying factors in photoperiodic control of the seasonal form in Polygonia c-aureum (Lepidoptera: Nymphalidae). Annot Zool Jpn 40:77-83

Hiroyoshi S (1997) Effects of photoperiod and age on the initiation of sperm movement in male Polygonia c-aureum $\mathrm{L}_{\text {INNAEus }}$ (Lepidoptera: Nymphalidae). Appl Entomol Zool 32:19-25

Hiroyoshi S (1999) Eupyrene and apyrene spermatogenesis in the Asian comma butterfly, Polygonia c-aureum (Lepidoptera: Nymphalidae). Entomol Sci 2:297-305

Hiroyoshi S (2000) Effects of aging, temperature and photoperiod on testis development of Polygonia c-aureum (Lepidoptera: Nymphalidae). Entomol Sci 3:227-236

Hiroyoshi S (2016) Effects of photoperiod on the testis fusion in the Asian comma butterfly, Polygonia c-aureum $\mathrm{L}_{\mathrm{INNAEUS}}$ (Lepidoptera: Nymphalidae). Entomon 41:159-168

Hiroyoshi S, Mitsuhashi J (1998) Effects of environmental conditions and aging on eupyrene sperm movement in male adults of Polygonia c-aureum (Lepidoptera: Nymphalidae). Zool Sci 15:561-565

Hodková A (1977a) Nervous pathways in photoperiodic regulation of reproduction in females of Pyrrhocoris apterus (Hemiptera). Acta Entomol Bohem 74:353-361

Hodková A (1977b) Function of the neuroendocrine complex in diapausing Pyrrchocoris apterus females. J Insect Physiol 23:23-28

Hoffmann KH, Behrens W (1982) Free ecdysteroids in adult male crickets, Gryllus bimaculatus. Physiol Entomol 7:269-279
Horn DH, Wilkie JS, Sage BA, O'Connor JA (1976) A high affinity antiserum specific for the ecdysone nucleus. J Insect Physiol 22:901-905

Ichimori T, Ohtomo R, Suzuki K, Kurihara M (1990) Specific protein related to adult diapause in the leaf beetle, Gastropysa atrocyanea. J Insect Physiol 36:85-91

Ismail PM, Ismail SM, Dutta-Gupta (Ray) A (1993) Ecdysteroid mediated uptake and secretion of larval haemolymph proteins by the male accessory reproductive glands of Chilo partellus. J Insect Physiol 10:811-815

Jindra M, Palli SR, Riddiford LM (2013) The juvenile hormone signaling pathway in insect development. Ann Rev Entomol $58: 181-204$

Kawasaki H, Kiguchi K, Agui N, Iwashit Y (1986) Ecdysteroid titer and wing development during the pupal-adult transformation of Bombyx mori. Zool Sci 3:301-308

Khalil Sayed MS, Anspaugh DD, Roe RM (2006) Role of juvenile hormone esterase and epoxide hydrolase in reproduction of the cotton bollworm, Helicoverpa zea. J Insect Physiol 52:669-678

Koolman J, Scheller K, Bodenstein D (1979) Ecdysteroids in the adult male blowfly Calliphora vicina. Experientia 35:134-135

Lemmen J, Keddie BA, Evenden ML (2016) Size and protein content of accessory glands in adult male Caloptiiia fraxinella in different physiological states. Physiol Entomol 41:74-82

Lessman CA, Herman WS, Schooley DA, Tsa LW, Bergot BJ, Baker FC (1989) Detection of juvenile hormone I, II and III in adult monarch butterflies (Danaus plexippus). Insect Biochem 19:431-433

Loeb MJ, Woods CW, Brandt EP, Borkovec AB (1982) Larval testes of the tobacco budworm: a new source of insect ecdysteroids. Science 218:896-898

Loeb MJ, Brandt EP, Birngbaum MJ (1984) Ecdysteroid production by testes of the tobacco budworm, Heliothis virescens, from last larval instar to adult. J Insect Physiol 30:375-381

Loeb MJ, Brandt EP, Woods CW, Bell RA (1988) Secretion of ecdysteroid by sheaths of testes of the gypsy moth, Lymantria dispar, and its regulation by testis ecdysiotropin. J Exp Zool 248:94-100

Loeb MJ, De Loof A, Gelman DB, Hakim RS, Jaffe H, Kochanskh JP, Meola SM, Schoofs L, Steel C, Vafopoulou X, Wagner RM, Woods CW (2001) Testis ecdysiotropin, an insect gonadotropin that induces synthesis of ecdysteroid. Archiv Insect Biochem Physiol 47:181-188

Marchal E, Verlinden H, Baduscio L, van Wielendaele P, Broeck JV (2012) RNAi-mediated knockdown of Shade negatively affects ecdysone-20-hydroxylation in the desert locust, Schistocerca gregaria. J Insect Physiol 58:890-896

Nishiitsutusji-Uwo J (1959) Developmental features of the male term cells in the prospective diapausing and non-diapausing swallowtails, Papilio xuthus. Mem Coll Sci Univ Kyoto Ser B 26:9-14

Numata H, Hidaka T (1984) Termination of adult diapause by a juvenile hormone analogue in the bean bug, Riptortus clavatus. Zool Sci 1:751-754

Pan ML, Wyatt GR (1971) Juvenile hormone induces vitellogenin synthesis in the monarch butterfly. Science 174:503-505

Paroulek M, Sláma K (2014) Production of the sesquiterpenoid, Juvenile Hormone-I (JH-I), and of vitamin E in the accessory sexual (colleterial) glands of adult male moths, Hyalophora cecropia (Linnaeus, 1758), (Lepidoptera: Saturniidae). Life 2:102-124

Parthasarathy R, Tan A, Sun Z, Chen J, Rainkin M, Palli SR (2009) Juvenile hormone regulation of male accessory gland activity in the red flour beetle, Tribolium castaneum. Mech Dev 126:563

Pener MP (1992) Environmental cues, endocrine factors, and reproductive diapause in male insects. Chronobiol Int 9:102-113

Pszczolkowski MA, Tucker A, Srinivasan A, Ramaswamy SB (2006) On the functional significance of juvenile hormone in the 
accessory sex glands of male Heliothis virescens. J Insect Physiol 52:786-794

Ram KR, Ramesh SR (2003) Male accessory gland proteins in Drosophila: a multifaceted field. Indian J Exp Biol 41:1372-1383

Ramalingam SE, Craig GB Jr (1977) Fine structure of the male accessory glands in Aedes triseriatus. J Insect Physiol 24:251-259

Richard DS, Watkikns NL, Serafin RB, Gilbert L-I (1998) Ecdysteroid regulate yolk protein uptake by Drosophila melanogaster oocytes. J Insect Physiol 44:637-644

Richard DS, Jones JM, Barbarito MR, Cerula S, Detweiler JP, Fisher SJ, Brannigan DM, Scheswohl DM (2001) Vitellogenesis in diapausing and mutant Drosophila melanogaster, further evidence for the relative roles of ecdysteroids and juvenile hormones. J Insect Physiol 47:905-913

Schooneveld H, Sanchez AO, de Wilde J (1977) Juvenile hormoneinduced break and termination of diapause in the Colorado potato beetle. J Insect Physiol 23:689-696

Seth RK, Kaur JJ, Rao DK, Reynolds SE (2004) Effects of larval exposure to sublethal concentrations of the ecdysteroid agonists RH-5849 and tebufenozide (RH-5992) on male reproductive physiology in Spodoptera litura. J Insect Physiol 50:505-517

Shiga S, Hamanaka Y, Tatsu Y, Okuda T, Numata H (2003) Juvenile hormone biosynthesis in diapause and nondiapause females of the adult blow fly Protophormia terraenovae. Zool Sci 20:1199-1206

Shimizu T (1989) Development of spermiduct and seminal vesicle during pharate adult of the cabbage armyworm, Mamestra brassicae (Lepidoptera: Noctuidae). Int Invertebr Reprod Dev 15:221-224

Shirk PD, Dahm KH, Röller H (1976) The accessory sex glands as the repository for juvenile hormone in the male Cecropia moths. Zeitsch Natur C 31:199-200

Shirk PD, Bhaskaran G, Roller H (1980) The transfer of juvenile hormone from male to female during mating in the Cecropia silkmoth Hyalophora cecropia. Experientia 36:682-683

Sláma K (2015) A new look at the nature of insect juvenile hormone with particular reference to studies carried out in the Czech Republic. Eur J Entomol 112:567-590

Socha R, Šula J, Kodrík D (2004) Wing morph-related differences in developmental pattern of accessory gland proteins in adult males of Pyrrhocoris apterus (L.) and their endocrine control. J Insect Physiol 50:893-901

Soller M, Bownes M, Kubli E (1999) Control of oocyte maturation in sexually mature Drosophila females. Dev Biol 208:337-351

Sridevi R, Bajaj P, Dutta-Gupta (Ray) A (1988) Ecdysteroid stimulated protein synthesis in the male accessory reproductive glands of Spodoptera litura. Invertebr Reprod Dev 14:177-186
Szopa TM, Rousseaux JL, Yuncker C, Happ GM (1985) Ecdysteroids accelerate mitosis accessory glands of beetle pupae. Develop Biol 107:325-336

Tauber MJ, Tauber CA, Masaki S (1986) Seasonal adaptation of insects. Oxford University Press, New York

Taub-Montemayor TE, Rankin MA (1997) Regulation of vitellogenin synthesis and uptake in the boll weevil, Anthonomus grandis. Physiol Entomol 22:256-260

Teal PEA, Gomez-Simuta Y, Proveaux AT (2000) Mating experience and juvenile hormone enhance sexual signaling and mating in male Caribbean fruit flies. PNAS 28:3706-3712

van Wielendaele P, Wynant N, Dillen S, Badisco L, Marchal E, Broeck JV (2013) In vivo effect of neuropeptide F on ecdysteroidogenesis in adult female desert locusts (Schistocerca gregaria). J Insect Physiol 59:624-630

Venkatesh K, Gillott C (1983) Protein production in components of the accessory gland complex of male Melanoplus sanguinipes (Insecta: Orhoptera). Inter J Invertebr Reprod 6:317-325

Wani M, Iwabuchi K, Agui N, Mitsuhashi J (1997) Endocrine alteration and precocious premetamorphic behaviors in the greater wax moth larvae, Galleria mellonella, parasitized by an endoparasitoid, Apanteles galleriae. Archiv Insect Biochem Physiol $34: 257-273$

Warren JT, Gilbert LI (1986) Ecdysone metabolism and distribution during the pupal-adult development of Manduca sexta. Insect Biochem 16:65-82

Wegener J, Huang ZY, Lorenz MW, Lorenz JI, Bienefeld K (2013) New insights into the roles of juvenile hormone and ecdysteroids in honey bee reproduction. J Insect Physiol 59:655-661

Wilson TG, De Moor S, Lei J (2003) Juvenile hormone involvement in Drosophila melanogaster male reproduction as suggested by the methoprene-tolerant mutant phenotype. Insect Biochem Mol Biol 33:1167-1175

Wu S-J, Zhan J-Z, Ma M (1987) Monitoring the effects of juvenile hormones and 20-hydroxyecdysone on yolk polypeptides production of Drosophila melanogaster with enzyme immunoassay. Physiol Entomol 12:355-361

Yaginuma T, Happ GM (1989) 20-hydroxyecdysone acts in the male pupa to commit accessory glands toward trehalase production in the adult mealworm beetle (Tenebrio molitor). Gen Comp Endocrin 73:173-185

Yaginuma T, Kai H, Happ GM (1988) 20-hydroxyecdysone accelerates the flow of cells into the $G_{1}$ phase and the $S$ phase in a male accessory gland of the mealworm pupa (Tenebrio molitor). Develop Biol 126:173-181 